\title{
Thermodynamic Database for Hydrogen Storage Materials
}

\author{
M.Baricco ${ }^{1, a}$, M.Palumbo $^{2, b}$, E.Pinatel ${ }^{1, c}$, M.Corno $^{1, \mathrm{~d}}$ and P.Ugliengo ${ }^{1, \mathrm{e}}$ \\ ${ }^{1}$ Dipartimento di Chimica I.F.M. and NIS, Università di Torino, via Giuria 7/9, 10125 Torino, Italy \\ ${ }^{2}$ STKS, ICAMS, Ruhr University Bochum, Stiepeler Str. 129, 44801 Bochum, Germany \\ amarcello.baricco@unito.it, ${ }^{\mathrm{b}}$ mauro.palumbo@rub.de, ${ }^{\mathrm{c}}$ eugenio.pinatel@unito.it, \\ dmarta.corno@unito.it, ${ }^{\mathrm{e}}$ piero.ugliengo@unito.it
}

Keywords: Hydrogen Storage, ab initio, computational thermodynamics, metallic and complex hydrides.

\begin{abstract}
In order to be used for applications, the thermodynamic stability of a candidate hydrogen storage material should be suitable for hydrogen sorption at room conditions. By mixing different hydrides, it is possible to promote the hydrogenation/dehydrogenation processes. On the other hand, small changes in composition allow a tailoring of thermodynamic stability of hydrides.

Knowledge of thermodynamic stability of hydrides is thus fundamental to study the hydrogenation/dehydrogenation processes and useful to rationalize synthesis reactions and to suggest possible alternative reaction routes.

The purpose of this work is to develop a consistent thermodynamic database for hydrogen storage systems by the CALPHAD approach. Experimental data have been collected from the literature. When experimental measurements were scarce or completely lacking, estimations of the energy of formation of hydrides have been obtained by ab initio calculations performed with the CRYSTAL code. Several systems of interest for hydrogen storage have been investigated, including metallic hydrides (M-H) and complex hydrides. The effect on thermodynamic properties of fluorine-tohydrogen substitution in some simple hydrides is also considered. Calculated and experimental thermodynamic properties of various hydrides have been compared and a satisfactory agreement has been achieved.
\end{abstract}

\section{Introduction}

Even if a suitable system design might improve significantly the properties of solid state hydrogen storage, intrinsic thermodynamic and kinetic properties of the material are fundamental.

Considering a generic hydrogenation reaction

$$
2 / x M+H_{2} \leftrightarrow 2 / x M H_{x}
$$

the equilibrium pressure $\left(p_{H}^{e q}\right)$ is given as a function of temperature $(T)$ by the Van't Hoff equation

$$
\ln p_{H_{2}}^{e q}=\frac{\Delta H_{0}}{R T}-\frac{\Delta S_{0}}{R}
$$

where $\Delta H_{0}$ and $\Delta S_{0}$ are the standard enthalpy and entropy of the reaction, and $\mathrm{R}$ is the gas constant. With a reference pressure $p_{H}^{e q}=1$ bar, the decomposition temperature $\left(T_{d e c}\right)$ can be calculated according to $\mathrm{T}_{\mathrm{dec}}=\Delta H_{0} / \Delta S_{0}$.

In reaction (1), $M H_{x}$ may represent a hydride phase of a pure component (e.g. $\mathrm{MgH}_{2}$ ), of an intermetallic compound (e.g. $\mathrm{LaNi}_{5} \mathrm{H}_{7}$ ) or of a combination of two elements (e.g. $\mathrm{NaBH}_{4}$ ). If $\Delta S_{0}=-130 \mathrm{~J} \cdot \mathrm{mol}_{\mathrm{H} 2}{ }^{-1} \mathrm{~K}^{-1}$ is considered, corresponding to the entropy change due to a mole of a gas which transforms into a solid [1], the Van't Hoff plot can be calculated for different values of $\Delta H_{0}$. 
An equilibrium at room conditions (i.e. $\mathrm{T}=300 \mathrm{~K}$ and $p^{e q}{ }_{H}=1$ bar) is obtained for $\Delta H_{0} \sim-40$ $\mathrm{kJ} \cdot \mathrm{mol}_{\mathrm{H} 2}{ }^{-1}$. If a constant pressure of 1 bar is fixed, a variation of $\Delta H_{0}$ of about $10 \mathrm{~kJ} \cdot \mathrm{mol}_{\mathrm{H} 2}{ }^{-1}$ shifts $T_{d e c}$ of about $100 \mathrm{~K}$. Similarly, if a constant temperature of $300 \mathrm{~K}$ is taken, a variation of $p^{e q}{ }_{H}$ of about two orders of magnitude is obtained for the same variation of $\Delta H_{0}$. It is then clear that small variations in $\Delta H_{0}$ can significantly modify the hydrogenation/deydrogenation conditions. In fact, the thermodynamic conditions for the hydrogen charge/discharge reactions can be tailored by a suitable selection of the crystal structure and the microstructure of the materials in the hydrogenated and dehydrogenated states.

\section{Modelling}

A full picture of the thermodynamic properties of a system can be obtained by the CALPHAD approach [2]. The goal is to obtain a description of the dependence of the free energy of all phases on temperature, pressure and composition. The analytical description of the temperature dependence of free energy, enthalpy, entropy and specific heat is given by parametric expressions [2]. The composition dependence can be described analytically by suitable models, such as the sublattice model, which contains interactions parameters [2]. For high order systems, several interpolation approaches have been suggested and, if necessary, high order interaction parameters can be introduced.

The interaction parameters are obtained by a least square procedure, starting from experimental values of existing phase diagrams and thermodynamic data. The base of the CALPHAD method is the availability of thermochemical data related to the investigated systems. In absence of experimental information, the output of various thermodynamic or quantum mechanical models can be used [3]. In particular, an estimation of the energy of formation of a compound can be obtained by ab initio calculations. Density Functional Theory (DFT) has proven to be very reliable when applied to predict structures and energetics of materials at the atomistic scale. As an example of the suggested methodology, ab initio calculations on $\mathrm{NaBH}_{4}$ have been recently reported [4].

It is customary to chose the pure solid elements in their stable phases at $298.15 \mathrm{~K}$ and $101325 \mathrm{~Pa}$ as reference state (SER, Standard Element Reference). The Gibbs free energy of the pure elements (lattice stability) is represented as a function of temperature in the form [2]:

$$
{ }^{0} G(T)=a+b T+c T \ln T+d T^{2}+e T^{3}+f / T+\sum_{\mathrm{n}} \mathrm{g}_{\mathrm{n}} T^{n}
$$

where $a$ to $f$ and $g_{n}$ are coefficients and $n$ represents a set of integers. The corresponding coefficients for unary systems were taken from the compilation of Dinsdale [6].

For condensed phases, the variation of the Gibbs free energy with pressure is negligible in the range of interest $(<10 \mathrm{MPa})$ [5]. Thus, pressure dependence of the Gibbs energy is only taken into account for the gas phase. An ideal gas behaviour has been assumed in the considered temperature and pressure range. The molar Gibbs energy of the gas phase is then given by:

$$
G^{\text {gas }}(T)=\sum_{i} x_{i}\left[{ }^{0} G_{i}^{\text {gas }}+R T \ln \left(P / P_{0}\right)\right]+R T \sum_{i} x_{i} \ln x_{i}
$$

where ${ }^{0} G_{i}^{\text {gas }}$ is the standard Gibbs energy of the component $i$ in the gas state, $P_{0}$ is the standard pressure of $101325 \mathrm{~Pa}, x_{i}$ is the mole fraction of species $i$ and $R$ is the gas constant. The Gibbs energy functions for the gaseous species were obtained from JANAF tables [7].

When no experimental evidence exists of a solubility range in hydrides, they can be considered as stoichiometric phases. Accordingly, the Gibbs free energy is expressed as follows: 


$$
G^{\mathrm{M}\left(\mathrm{XH}_{\mathrm{z}}\right)_{\mathrm{y}}}=x_{X}{ }^{0} G_{X}+x_{H}{ }^{0} G_{H}^{g a s}+x_{M}{ }^{0} G_{M}+\Delta H_{f o r}-T \cdot \Delta S_{f o r}
$$

where $\Delta H_{f o r}$ and $\Delta S_{f o r}$ have been assessed on the basis of ab initio and/or experimental results.

All thermodynamic calculations in this work have been performed using the ThermoCalc software package and the PARROT optimisation module. The CRYSTAL code has been used for DFT calculations $[8,9]$.

\section{Metallic Hydrides}

Several systems involving metallic hydrides have been thermodynamically investigated. For example, the Na-H [10], Li-H [11], Fe,Sm-H [5], Mg,Ni-H [12], Al-H [13], Cu,Pd-H [14], Ti,Zr-H [15], Pd-H-D-T [16] systems have been thermodynamically assessed by different authors. Recently, the La-H system has been evaluated [17] and it will be described in the following as a model for metal-hydrogen system.

One binary $\mathrm{LaH}_{\mathrm{x}}$ hydride has been reported in the La-H system with an f.c.c. structure (space group Fm-3m). Usually, La atoms occupy the 4a sites, while hydrogen atoms occupy the tetrahedral $8 \mathrm{c}$ and octahedral $4 \mathrm{~b}$ sites. Hydrogen atoms occupy tetrahedral sites first, for concentration up to $\mathrm{x}=1.95$ and, above this concentration, octahedral sites begin to be occupied. Accordingly, a 3 sublattice model $(\mathrm{La})_{1}(\mathrm{H}, \mathrm{Va})_{2}(\mathrm{H}, \mathrm{Va})_{1}$ has been applied to describe the Gibbs free energy of this hydride [17], the second sublattice corresponding to tetrahedral sites while the third sublattice corresponds to the octahedral sites.

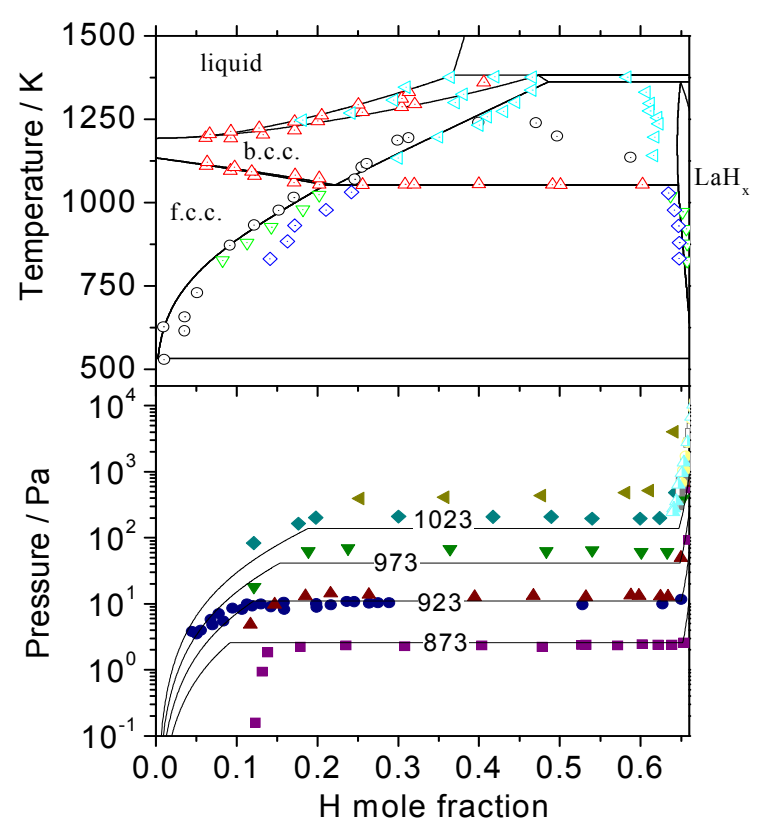

Fig. 1 - Above: calculated La-H phase diagram (continuous line) together with experimental points. Below: calculated P-C-T curves for the La-H system (continuous lines, temperature indicated in $\mathrm{K}$ ) together with experimental points. Experimental points from ref. [17].

The calculated phase diagram according to present assessment is reported in Fig. 1. A good agreement has been obtained between experimental data and calculated phase boundaries. The eutectoid point corresponding to the reaction b.c.c. $\rightarrow$ f.c.c. $+\mathrm{LaH}_{\mathrm{x}}$ is calculated at $1053 \mathrm{~K}$ in $\operatorname{good}$ agreement with the experimental value equal to $1051 \mathrm{~K}$. The eutectoid point at lower temperature (f.c.c. $\rightarrow$ d.h.c.p. $+\mathrm{LaH}_{\mathrm{x}}$ ) is also in satisfactory agreement with the experiments. The present results confirm that no miscibility gap occurs in the b.c.c. phase and equilibria between b.c.c. and $\mathrm{LaH}_{\mathrm{x}}$ phases are calculated up to $1363 \mathrm{~K}$, where the decomposition of the binary hydride occurs into b.c.c. and gas phase. In Fig. 1, calculated p-c-T curves are compared with experimental determinations at several temperatures. The agreement between experimental data and calculated results in the considered temperature range is satisfactory, with the highest deviation occurring at lower temperatures.

In comparison to simple metal hydrides $\mathrm{M}-\mathrm{H}$, thermodynamic evaluations related to hydrides

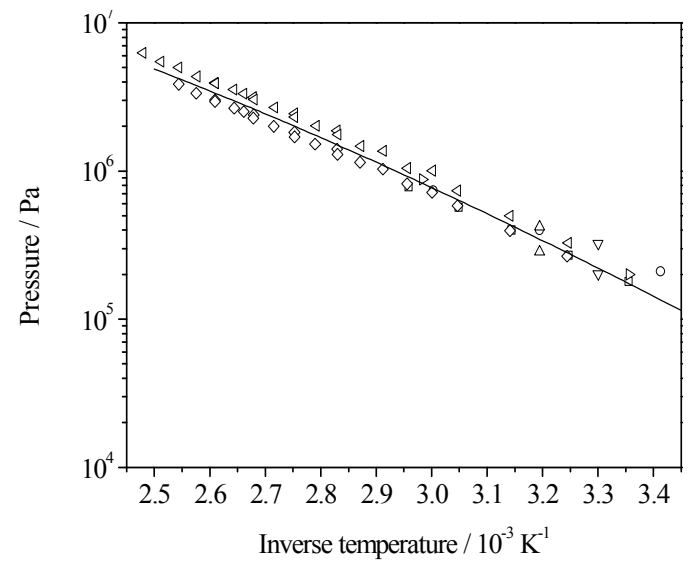

Fig. 2 - Calculated Van't Hoff plot for $\mathrm{LaNi}_{5}$ compound (continuous line) together with experimental points. Experimental points from ref. [17]. 
formed from intermetallic compounds are more limited. Among the few systems investigated, there are the $\mathrm{Mg}-\mathrm{Ni}-\mathrm{H}$ system with $\mathrm{Mg}_{2} \mathrm{NiH}_{4}$ and $\mathrm{Mg}_{2} \mathrm{NiH}_{1-x}$ hydrides [18], and the ternary La-Ni-H system with the $\mathrm{LaNi}_{5} \mathrm{H}_{7}$ hydride [17]. The last system is of importance since commercial applications of $\mathrm{LaNi}_{5}$ compound as an hydrogen storage materials are already available. Fig. 2 reports the calculated van't Hoff plot for the ternary $\mathrm{LaNi}_{5} \mathrm{H}_{7}$ hydride. Several data sets are also shown for comparison and the agreement obtained is good. It must be pointed out that p-c-T measurements are greatly affected by sample preparation, conditions and activation. During optimization and in Fig. 2, a selection of p-c-T data has been used, whose conditions are closer to thermodynamic equilibrium [17].

\section{Complex Hydrides}

Complex hydrides based on light elements have been extensively investigated [1]. However, thermodynamic and kinetic properties of these materials still present some drawbacks which hinder their commercial application as hydrogen storage materials. Alanate systems have been widely investigated in recent years, and assessed data on $\mathrm{Na}_{3} \mathrm{AlH}_{6}, \mathrm{NaAlH}_{4}, \mathrm{Li}_{3} \mathrm{AlH}_{6}, \mathrm{LiAlH}_{4}$ have been reported [19,20]. The $\mathrm{Mg}\left(\mathrm{AlH}_{4}\right)_{2}$ alanate has a rather high theoretical hydrogen storage capacity, but its thermodynamic properties are unsuitable for hydrogen storage, as it has been shown by a combined ab initio/ thermodynamic approach [21].

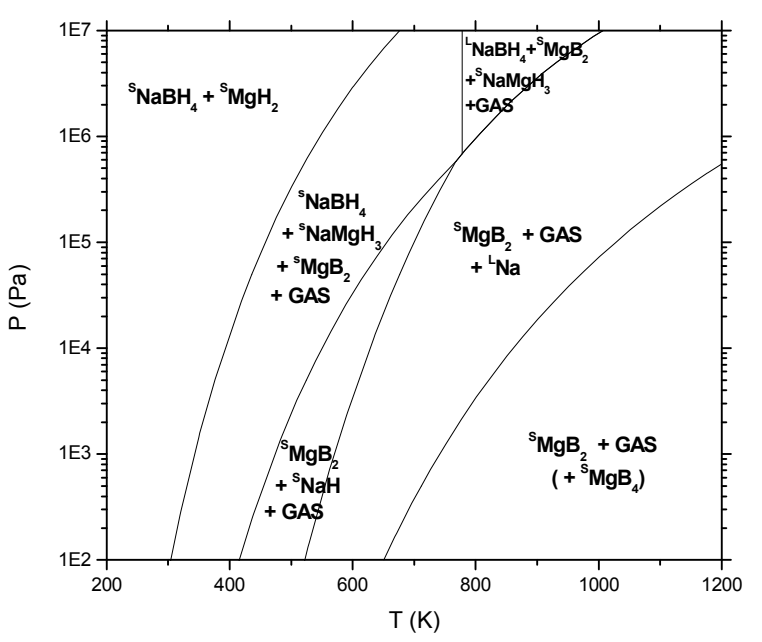

Fig. 3 - Calculated pressure vs. temperature phase diagram for $2 \mathrm{NaBH}_{4}+\mathrm{MgH}_{2}$

In order to bring the temperature and pressure of hydrogen absorption/desorption close to ambient conditions in complex hydrides, Reactive Hydride Composites (RHC) have been suggested [22]. They consist of two hydrides mixed together by ball milling, which allow a significant reduction of the reaction enthalpy. The dehydrogenation pathway in the $2 \mathrm{NaBH}_{4}+\mathrm{MgH}_{2}$ composite was experimentally studied, making use of complementary characterization techniques. It was found that dehydrogenation reaction is likely to proceed as follows [23]: $2 \mathrm{NaBH}_{4}+\mathrm{MgH}_{2}\left(>300{ }^{\circ} \mathrm{C}\right) \rightarrow$ $2 \mathrm{NaBH}_{4}+1 / 2 \mathrm{MgH}_{2}+1 / 2 \mathrm{Mg}+1 / 2 \mathrm{H}_{2}\left(>350^{\circ} \mathrm{C}\right) \rightarrow 3 / 2 \mathrm{NaBH}_{4}+1 / 4 \mathrm{MgB}_{2}+1 / 2 \mathrm{NaH}+3 / 4 \mathrm{Mg}+$ $7 / 4 \mathrm{H}_{2}\left(>450{ }^{\circ} \mathrm{C}\right) \rightarrow 2 \mathrm{NaH}+\mathrm{MgB}_{2}+4 \mathrm{H}_{2}\left(>500{ }^{\circ} \mathrm{C}\right) \rightarrow 2 \mathrm{Na}+\mathrm{MgB}_{2}+5 \mathrm{H}_{2}$. Furthermore, the presence of an intermediate phase $\left(\mathrm{NaMgH}_{3}\right)$ suggests the occurrence of secondary reactions with low kinetics, which may slow down the main reaction.

In order to rationalize these results, Fig. 3 shows the calculated $\mathrm{P}$ vs. $\mathrm{T}$ phase diagram corresponding to the composition $2 \mathrm{NaBH}_{4}+\mathrm{MgH}_{2}$. The free energy parameters for $\mathrm{NaMgH}_{3}$ were obtained on the basis of experimental data for $\Delta H_{f o r}$ and $\Delta S_{f o r}$ [24]. In the calculated phase diagram, the formation of $\mathrm{NaMgH}_{3}$ is predicted as thermodynamically stable in a certain P-T range. A sluggish kinetics could explain why this mixed hydride is not observed experimentally during the first stages of thermal dehydrogenation. The presence of $\mathrm{NaH}, \mathrm{Na}$ and $\mathrm{MgB}_{2}$ is predicted at higher temperatures, according to experimental findings [23].

\section{Fluorine Substituted Hydrides}

The substitution of hydrogen with fluorine in complex hydrides, aimed to a thermodynamic destabilization of the compound, has been explored in alanate systems [25]. Experimental results have been compared with $a b$ initio calculations of the energy of formation as a function of the $\mathrm{F} / \mathrm{H}$ ratio. The fluoride addition results in a substitution of $\mathrm{H}^{-}$by $\mathrm{F}^{-}$anion in the hydride lattice and, accordingly, a favorable thermodynamics adjustment. The coupled computational and experimental 
efforts allowed to put forward a functional anion concept [26]. An estimation of the thermodynamics of the RHC has been carried out considering a wide number of systems [27], including fluorides. As an example, it has been reported that ball milling $\mathrm{LiF}$ with $\mathrm{MgH}_{2}$ gives a mixture that can be easily hydrogenated (at 100 bar), leading to $\mathrm{LiBH}_{4}$ and $\mathrm{MgF}_{2}$, even if the reaction appears only partially reversible [28].

A coupled $a b$ initio/CALPHAD study has been carried out to investigate the thermodynamic behaviour of the $\mathrm{AlH}_{3}-\mathrm{AlF}_{3}$ and $\mathrm{MgH}_{2}-\mathrm{MgF}_{2}$ solid solutions. Ab initio calculations based on DFT GGA Hamiltonian (PBE) have been carried out with the periodic CRYSTAL06 [8,9] code. Phonons at Gamma point in the harmonic approximation have been computed to derive the thermodynamic functions. When an atom of hydrogen is substituted inside the cell, it can be substituted in different positions leading to different configurations not always equivalent by

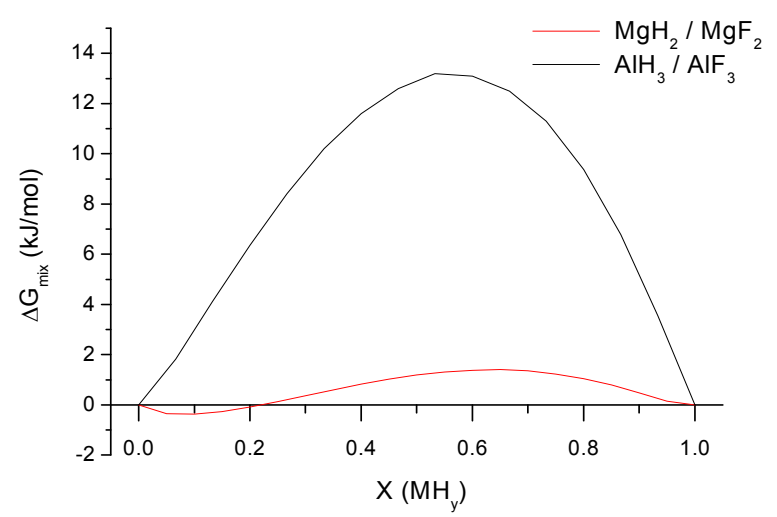

Fig. 4 - Energy of mixing for the $\mathrm{MF}_{\mathrm{y}}-\mathrm{MH}_{\mathrm{y}}(\mathrm{M}=\mathrm{Al}, \mathrm{y}=3$ and $\mathrm{M}=\mathrm{Mg}, \mathrm{y}=2$ ) solid solutions as function of composition at $298 \mathrm{~K}$. symmetry. By the use of a newly implemented algorithm within the CRYSTAL code [29] each configuration is automatically screened on a symmetry base, so that the calculations are reduced only to the irreducible configurations. For each composition, only the most stable configuration was considered in the thermodynamic assessment. In the case of the $\mathrm{AlH}_{3}-\mathrm{AlF}_{3}$ solid solution, five compositions were computed, and the corresponding thermodynamic values have been used to calculate $\Delta \mathrm{H}, \Delta \mathrm{S}$ and $\Delta \mathrm{G}$ of mixing for the reaction (1-a) $\mathrm{AlH}_{3}+a \mathrm{AlF}_{3} \rightarrow \mathrm{AlH}_{3-3 a} \mathrm{~F}_{3 a}$ with $\mathrm{a}=1 / 6,1 / 3,1 / 2,2 / 3$ and $5 / 6$ at $\mathrm{T}=298 \mathrm{~K}$ and $\mathrm{p}=101325 \mathrm{~Pa}$.

In Fig. 4 the calculated free energy of mixing for the $\mathrm{AlH}_{3}-\mathrm{AlF}_{3}$ and $\mathrm{MgH}_{2}-\mathrm{MgF}_{2}$ solid solutions is reported as function of composition at $298 \mathrm{~K}$. For the $\mathrm{MgH}_{2}-\mathrm{MgF}_{2}$ solution the mixing energy is positive at room temperature for almost every composition and only in the fluorine rich side a small solubility of hydrogen is predicted. The higher free energy of mixing of the $\mathrm{AlH}_{3}-\mathrm{AlF}_{3}$ solid solution instead prevents the formation of a unique phase for all compositions.

\section{Conclusions}

CALPHAD thermodynamic models are available for a proper description of the Gibbs free energy of hydrogen absorption as a function of temperature, pressure and composition. Accurate experimental data are needed and ab initio estimation can also be used when data are lacking. Reliable thermodynamic databases can be obtained for multicomponent systems by the assessment procedure and thermodynamic results can be used as input parameters in several kinetic models.

\section{Acknowledgments}

This work has been supported by Euroepan Union under the contracts COSY and FLYHY.

\section{References}

[1] A. Züttel: Naturwissenschaften Vol. 91 (2004), p.157

[2] B. Sundman, S.G. Fries and H.L. Lukas: Computational Thermodynamics (Cambridge 2007)

[3] P.E.A. Turchi, I.A. Abrikosov, B. Burton, S.G. Fries, G. Grimvall, L. Kaufman, P. Korzhavyi, V.R. Manga, M. Ohno, A. Pisch, A. Scott and W. Zhang: CALPHAD Vol. 31 (2007), p. 4 
[4] J. Urgnani, F.J.Torres, M. Palumbo and M. Baricco: Int. J. Hydr. Energy Vol. 33 (2008), p.3111

[5] M. Zinkevich, N. Mattern, A. Handstein and O. Gutfleisch: J. Alloys Compd Vol. 339 (2002), p. 118

[6] A.T. Dinsdale: CALPHAD Vol. 15 (1991), p. 317

[7] M.W. Chase Jr., NIST-JANAF Thermochemical Tables, fourth ed., J. Phys. Chem. Ref. Data Monograph No. 9

[8] R. Dovesi, V. R. Saunders, C. Roetti, R Orlando, C. M. Zicovich-Wilson, F. Pascale, B. Civalleri, K. Doll, N.M. Harrison, I.J. Bush, P. D’Arco, M. Llunell, CRYSTAL2006 User's Manual; http://www.crystal.unito.it, University of Torino: Torino, 2006.

[9] R. Dovesi, R. Orlando, B. Civalleri, C. Roetti, V.R. Saunders and C.M. Zicovich-Wilson: Z. Kristallogr. Vol. 220 (2005), p. 571

[10] C. Qiu, G.B. Olson, S.M. Opalka and D.L. Anton: Int. J. Mat. Res. Vol. 97 (2006), p. 845

[11]A.D. Pelton: Z. Melallkde. Vol. 84 (1993), p. 767

[12] K. Zeng, T. Klassen, W. Oelerich and R. Bormann: Int. J. Hydrogen Energy Vol. 24 (1999), p. 989

[13] C. Qiu, G.B. Olson, S.M. Opalka and D.L. Anton: J. Phase Equilib. Diff. Vol. 25 (2004), p. 520

[14] W. Huang, S.M. Opalka, D. Wang and T.B. Flanagan: CALPHAD Vol. 31 (2007), p. 315

[15] E. Konigsberger, G. Eriksson and W.A. Oates: JALCOM Vol. 299 (2000), p. 148

[16] J.-M Joubert and S. Thiébaut: Journal of Nuclear Materials Vol. 395 (2009), p. 79

[17] M. Palumbo, J. Urgnani, D. Baldissin, L. Battezzati and M. Baricco: CALPHAD Vol. 33 (2009), p. 162

[18]K. Zeng, T. Klassen, W. Oelerich and R. Bormann: JALCOM Vol. 299 (1999),p. 213

[19] B.-M. Lee, J.-W. Jang, J.-H. Shimb, Y.W. Cho and B.-J. Lee: JALCOM Vol. 424 (2006), p. 370

[20] J.-W. Jang, J.-H. Shimb, Y. W. Cho and B.-J. Lee: JALCOM Vol. 420 (2006), p. 286

[21] M. Palumbo, F.J. Torres, J.R. Ares, C. Pisani, J.F. Fernandez and M. Baricco, CALPHAD Vol. 31 (2007), p. 457.

[22] J. Vajo, S.L. Skeith and F. Mertens: J Phys. Chem. B Lett. Vol. 109 (2005), p. 3719

[23]D. Pottmaier, E. Groppo, S. Bordiga, G. Spoto and M. Baricco: Proc. HYSYDAYS 2009, Turin, Italy, Oct. 2009.

[24] K. Ikeda, S. Kato, Y. Shinzato, N. Okuda, Y. Nakamori, A. Kitano, H. Yukawa, M. Morinaga and S. Orimo: Journal of Alloys and Compounds Vol. 446-447 (2007), p. 162

[25]P.Wang, X.D.Kang and H.M.Cheng: ChemPhysChem Vol. 6 (2005), p. 2488

[26] L.Yin, P.Wang, X.Kang, C.Sun and H.Cheng: Phys. Chem. Chem. Phys. Vol. 9 (2007), p. 1499

[27] J.J. Vajo and G.L. Olson : Scripta Mat. Vol. 56 (2007), p. 829

[28] J.J. Vajo, T.T. Salguero, A.F. Gross, S.L. Skeith and G.L. Olson, J. All. Comp. Vol. 446-447 (2007) p. 409

[29]A. Meyer, P. D'Arco, R. Orlando and R. Dovesi: J. Phys. Chem. C Vol.113(2009), p. 14507 